Review began 09/09/2021 Review ended 02/13/2022 Published 02/22/2022

๑) Copyright 2022

Jain et al. This is an open access article distributed under the terms of the Creative Commons Attribution License CC-BY 4.0. which permits unrestricted use, distribution, and reproduction in any medium, provided the original author and source are credited.

\section{Pyrexia Unmasking Brugada Syndrome: A Literature Review}

\author{
Ashish Jain ${ }^{1}$, K Yagnik ${ }^{2}$, Khandakar M. Hussain ${ }^{1}$, Sarvesh Naik ${ }^{1}$, Tanvi Sharma ${ }^{3}$, Asna Shahab ${ }^{1}$, \\ Muhammad Haroon Khilan ${ }^{1}$
}

1. Internal Medicine, Conemaugh Memorial Medical Center, Johnstown, USA 2. Medicine and Surgery, Government Medical College, Surat, IND 3. Medicine, School of Medical Sciences and Research, Greater Noida, IND

Corresponding author: Ashish Jain, drashishjain93@gmail.com

\begin{abstract}
Brugada syndrome $(\mathrm{BrS})$ is an inherited arrhythmia syndrome in which asymptomatic patients tend to develop fatal arrhythmias leading to sudden cardiac death (SCD) in asymptomatic or undiagnosed cases. This review tries to shed light on pyrexia being one of the triggers to cause SCD secondary to fatal arrhythmias in patients of BrS. Pyrexia, electrolyte imbalance, alcohol intake, and drugs are common triggering factors for fatal arrhythmias in patients with BrS. Most patients are asymptomatic, while the most common form of presentation that brings the patient under medical attention is syncope or SCD. Hence, patients, especially young, who present with syncope or aborted episode of SCD with typical EKG patterns, should undergo further workup. It is essential to educate patients about the condition, possible triggers, and the importance of refraining them.
\end{abstract}

Categories: Cardiology, Internal Medicine

Keywords: syncope, sudden cardiac death, brs, congenital cardiac arrhythmia syndrome, brugada syndrome

\section{Introduction And Background}

Brugada syndrome $(\mathrm{BrS})$ is an inherited cardiac arrhythmia syndrome with a polygenic mode of inheritance with variable expression and many common and rare genetic variants. It causes an increased risk for ventricular tachyarrhythmia and sudden cardiac death (SCD). It is characterized by a typical ECG pattern with pseudo-right bundle branch block and persistent ST-segment elevation in the right precordial leads (V1-V3) [1], first described in 1992 by a report by two Spanish doctors "Pedro and Joseph Brugada" who identified the pattern in eight individuals who were resuscitated from SCD caused by ventricular fibrillation (VF). Initially characterized as a right bundle branch block, persistent ST-segment elevation, and SCD syndrome, it was later renamed BrS. There is a reduction in the number or function of sodium ( $\mathrm{Na}+$ ) channels leading to a decreased sodium $(\mathrm{Na}+)$ current, which leads to a characteristic ECG pattern, while most of the patients stay asymptomatic. It has been shown to unmask itself in suspected individuals and is influenced by several external factors, including electrolyte imbalances, vagal tone, fever, and drugs (Figure 1). Several cases of BrS have been reported with pyrogenic conditions, which include infectious and noninfectious causes. Recently, various cases have been reported to be associated with pyrexia of coronavirus disease 2019 (COVID-19). Diagnosis of BrS is made by a typical spontaneous ECG presentation or a drug provocation test using a sodium channel blocker. The first line of management for $\mathrm{BrS}$ remains an implantable cardioverter-defibrillator (ICD), and other modalities such as radiofrequency ablation have shown to be effective in patients with contraindications for ICD. Asymptomatic patients or patients with ventricular premature beats or unsustained ventricular tachycardia with typical ECG findings are said to have a Brugada pattern. In contrast, patients with characteristic ECG findings who have experienced SCD or sustained ventricular tachycardia have BrS. This review focuses on the clinical presentation, diagnosis, riskstratification, and management of pyrexia-induced BrS [2]. 


\section{Cureus}

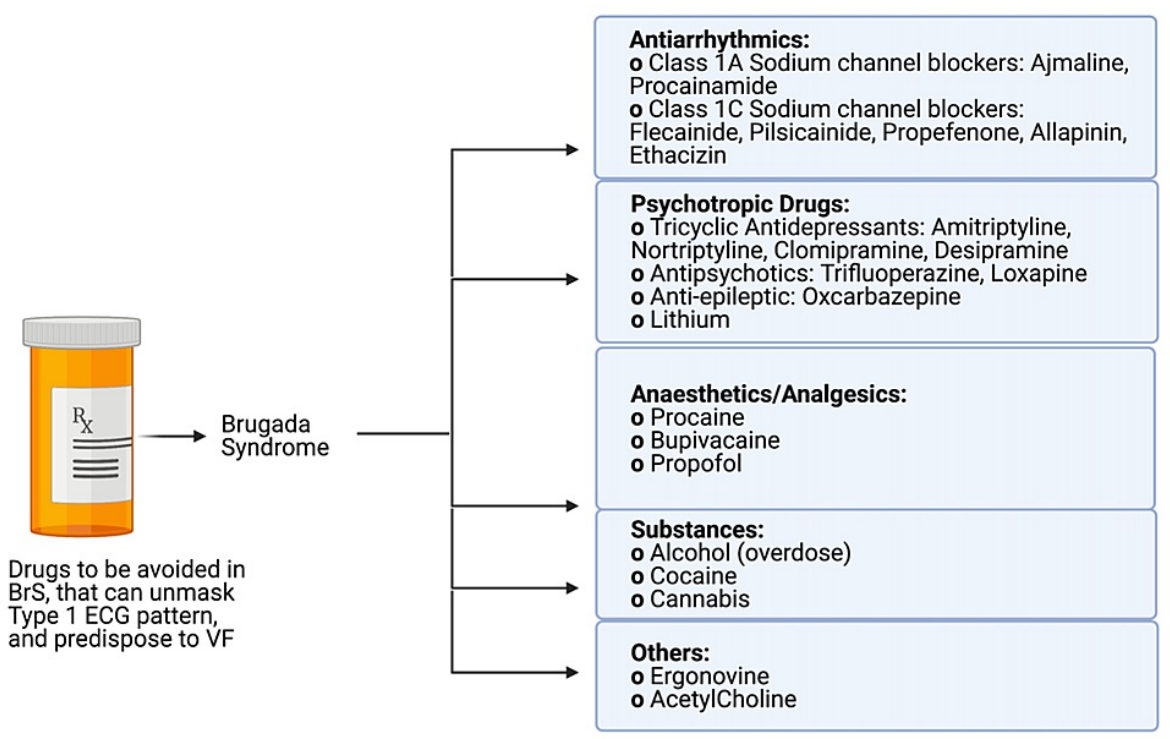

FIGURE 1: Drugs known to unmask BrS pattern on ECG and predispose to VF.

Created with BioRender.com

$\mathrm{BrS}=$ Brugada syndrome; $\mathrm{VF}=$ ventricular fibrillation

\section{Review}

\section{Clinical presentation}

One-third of BrS patients are identified after evaluating symptoms such as syncope or an aborted episode of SCD [3], and spontaneous atrial fibrillation can be seen in approximately $13 \%$ of patients with $\mathrm{BrS}$, suggesting an abnormality in both atria and ventricle [4]. Brugada pattern can be exhibited by a variety of diseases and conditions, including myocardial ischemia, pulmonary embolism, right ventricular compression, acute pericarditis, and electrolyte imbalances (hypokalemia, hyperkalemia, and hypercalcemia). These Brugada pattern phenotypes on ECG can result in a misdiagnosis and should be evaluated systematically, which includes electrolyte levels, cardiac markers, and imaging, such as echocardiography and CT chest with/without contrast. Pyrexia with an infectious or non-infectious etiology is a trigger known to induce fatal arrhythmias such as VF in diagnosed as well as asymptomatic/undiagnosed cases of BrS. It is critical to keep BrS as a differential diagnosis in a patient presenting with syncope, VF, or sudden cardiac arrest/death with a recent history of fever [5]. Several cases of pyrexia associated with COVID-19 have been associated with unmasking silent BrS and are mentioned below (Table 1).

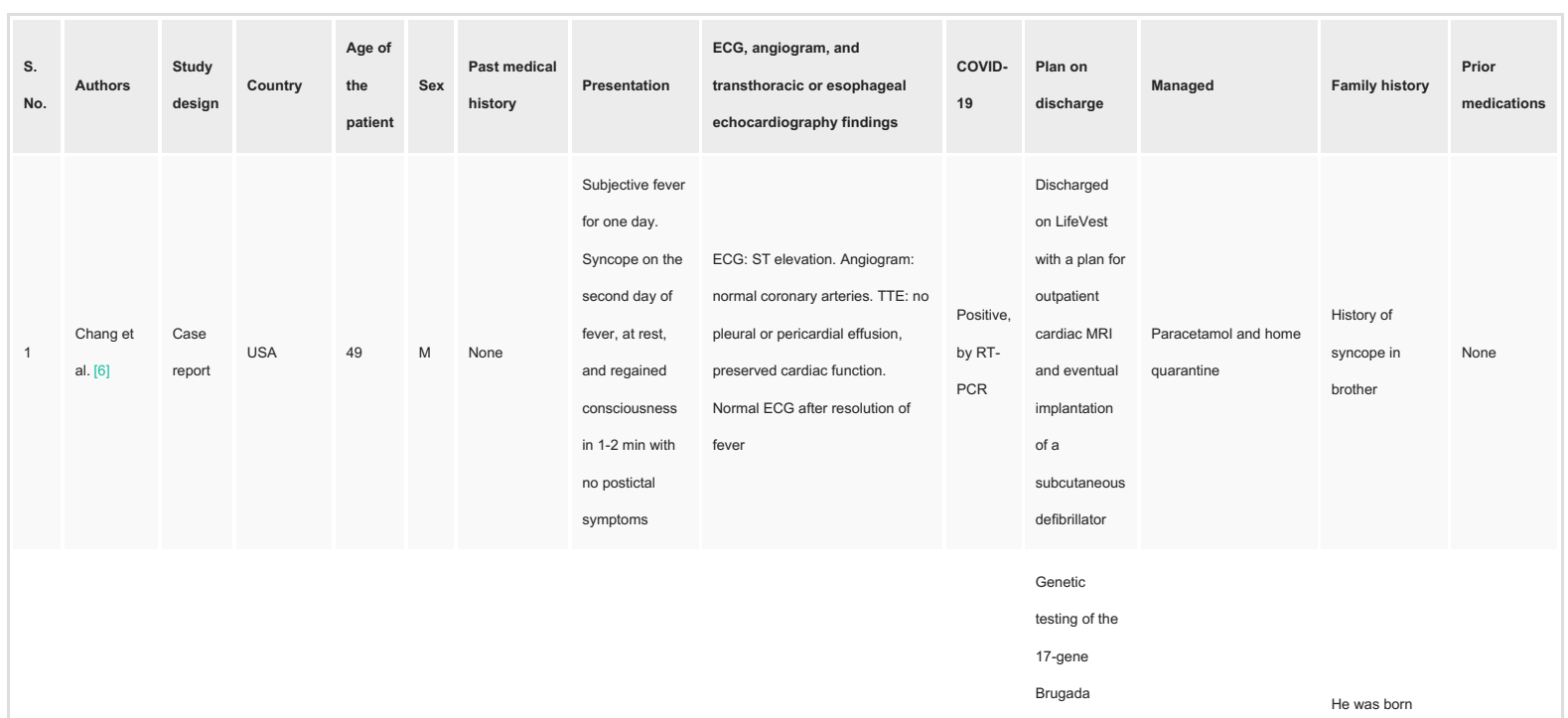




\section{Cureus}

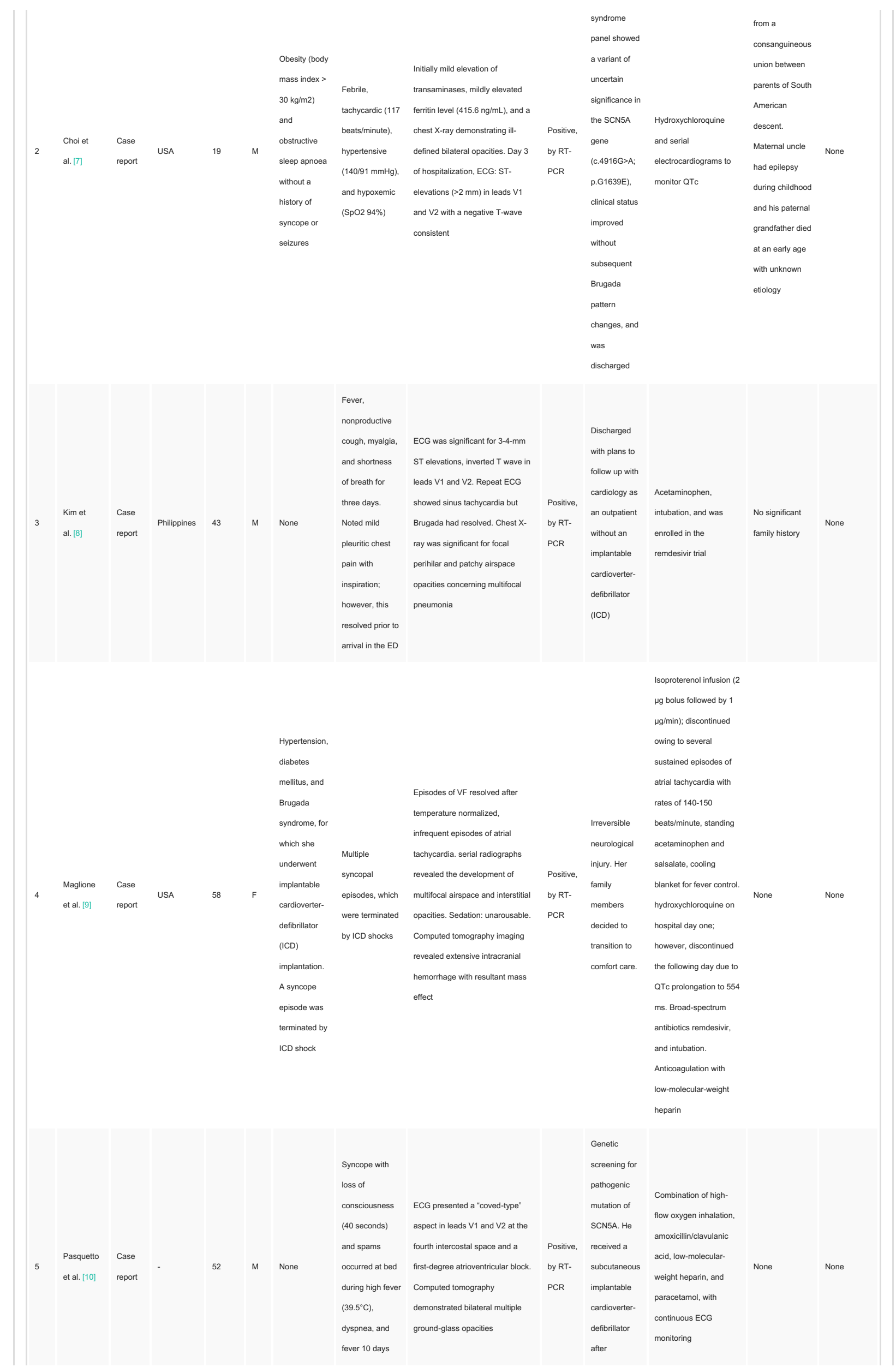




\section{Cureus}

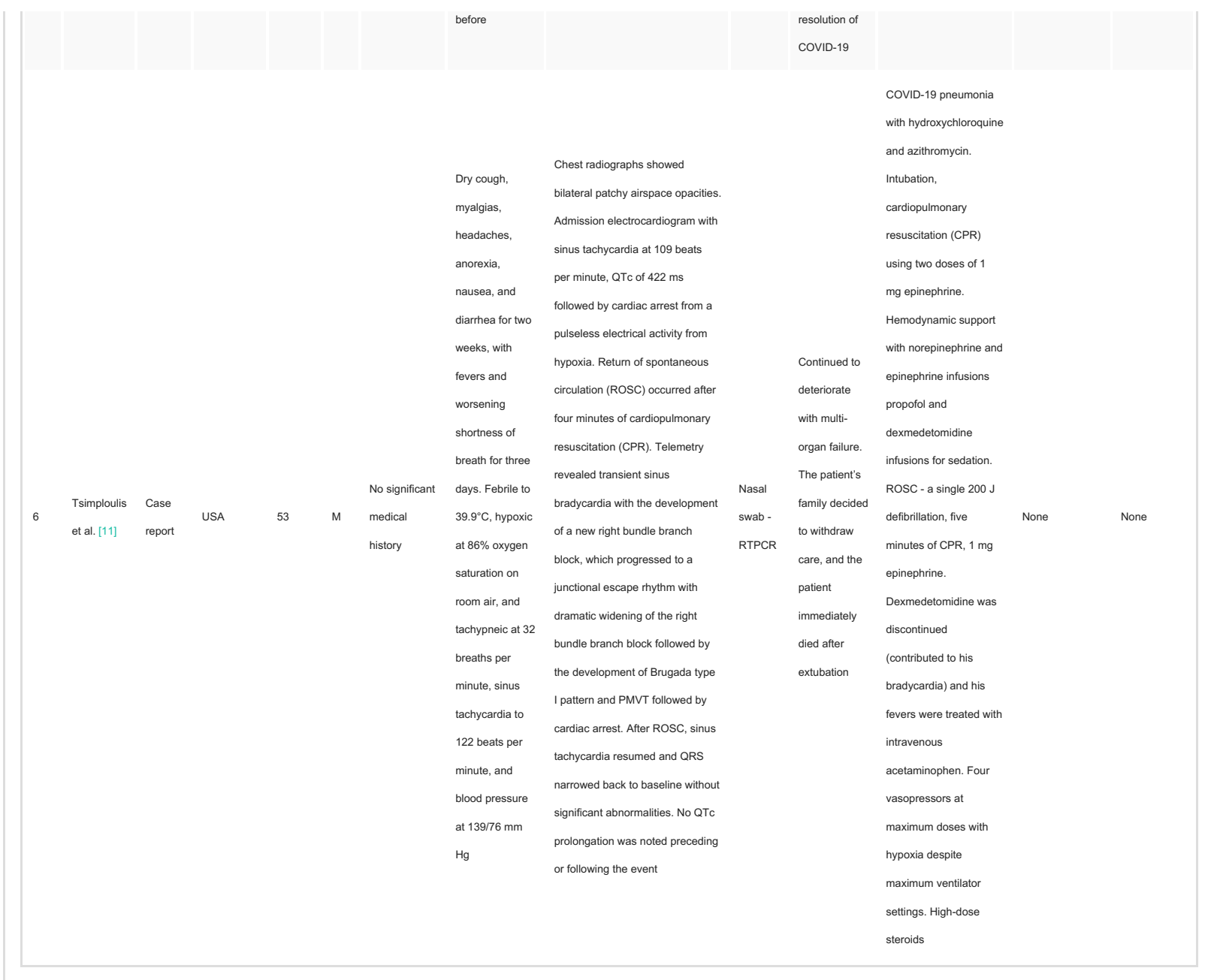

TABLE 1: Recent case reports on patients of COVID-19 with BrS.

COVID-19 = coronavirus disease 2019; ECG = electrocardiogram; ED = emergency department; ICD = implantable cardioverter-defibrillator; TTE = transthoracic echocardiography; VF = ventricular fibrillation; ROSC = return of spontaneous circulation; RT-PCR = reverse transcription-polymerase chain reaction; CPR = cardiopulmonary resuscitation; SpO2 = oxygen saturation; SCN5A = sodium voltage-gated channel alpha subunit 5 ; QTC = corrected $\mathrm{QT} ; \mathrm{PMVT}=$ polymorphic ventricular tachycardia.

\section{Diagnosis}

A diagnosis of BrS is based on both clinical and electrophysiological parameters. A typical ECG pattern, which was previously known as type 1 ECG with "coved ST-segment elevation" of $>2 \mathrm{~mm}$ in the precordial leads ending with a negative T wave, is required to diagnose patients with or without symptoms. Patterns apart from the typical ECG findings of BrS cannot confirm the diagnosis and need to be further evaluated by sodium channel blocker test (flecainide ( $2 \mathrm{mg} / \mathrm{kg}$ over 10 minutes) and procainamide or ajmaline $(1 \mathrm{mg} / \mathrm{kg}$ over 5-10 minutes)). Ajmaline has been shown to be more sensitive than flecainide or procainamide in inducing type 1 patterns [12]. A postprandial or full stomach test increases vagal tone and is another diagnostic modality that can also be used to unmask type 1 ECG patterns. The risk of conversion to ventricular arrhythmia is low if the sodium channel blocker provocation test is done under proper supervision and by experienced individuals.

Type 1 (Coved Type)

At least one of the right precordial leads (V1-V3) showing an ST elevation greater than or equal to $2 \mathrm{~mm}$ (J wave amplitude) gives rise to a gradually descending ST segment-terminal portion, followed by a negative $\mathrm{T}$ wave with little or no isoelectric separation (Figure 2) [13]. 


\section{Cureus}

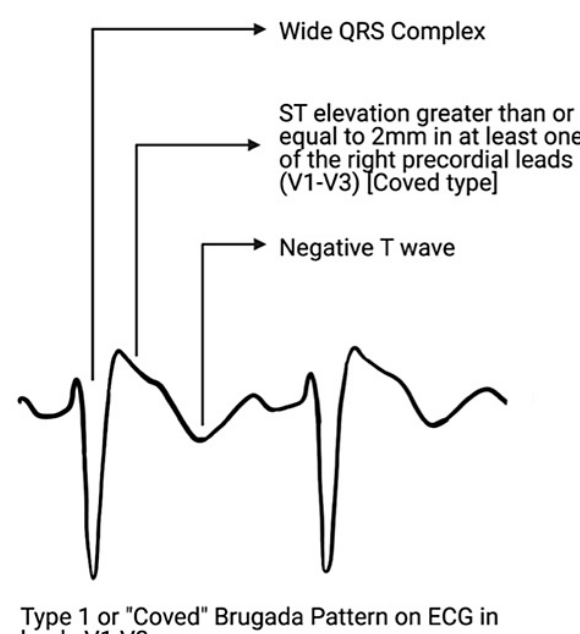

Type 1 or "Coved" Brugada Pattern on ECG in
leads V1-V3

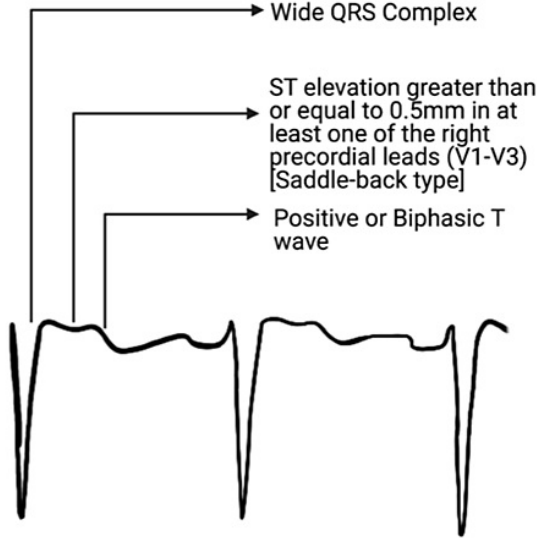

Type 2 or "Saddle-back"Pattern on ECG in leads V1-V3

FIGURE 2: A diagrammatic representation of type 1 and type 2 Brugada pattern on EKG.

Type 2 (Saddle-Back Type)

At least one of the right precordial leads (V1-V3) showing an ST elevation greater than or equal to $2 \mathrm{~mm}$ (J wave amplitude) gives rise to gradually descending ST segment-terminal portion (remaining $\geqslant 1 \mathrm{~mm}$ above the baseline), and a subsequent positive or biphasic T wave (Figure 2) [13,14].

Type 3 (Saddle-Back Type)

At least one of the right precordial leads (V1-V3) showing an ST elevation greater than or equal to $2 \mathrm{~mm}$ (J wave amplitude) gives rise to descending ST segment-terminal portion (remaining $<1 \mathrm{~mm}$ above the baseline), followed by positive T wave [13].

Genetic screening in clinical practice may be restricted to the population at risk, i.e., genetically related family members of a diagnosed patient. Over 20 genes have been identified to be associated with BrS. The sodium voltage-gated channel alpha subunit 5 (SCN5A) gene encodes the alpha subunit of the main cardiac sodium channel $(\mathrm{Na}(\mathrm{v}) 1.5)$, which maintains the inward sodium current and plays a vital role in regulating cardiac electrophysiological function. SCN5A gene and its more than 300 variants are associated with a quarter of cases diagnosed with BrS, these are usually loss of function variants, including non-sense variants, missense variants, nucleotide deletion/insertion variants, and splice site variants with the observation of incomplete penetrance among the carriers of the mutation.

\section{Risk stratification}

A study conducted by Priori et al. in 2013 stratified patients based on the risk of cardiac arrest, and based on their finding, patients with baseline ST elevation with syncope are the highest risk category and require an ICD placement while patients with ECG changes and no symptoms were categorized as intermediate risk and patients with ECG changes only after drug provocation were classified as low risk. The incremental predictive usefulness of an electrophysiology study (EPS) based on clinical factors is highly debatable. While some writers asserted a link between induced VF and cardiac events, extensive prospective investigations have established that an EPS does not accurately stratify the risk of arrhythmia. The most recent recommendations limit an EPS for ICD implantation to a class IIb $[15,16]$.

COVID-19 pandemic has led to hospital rush, creating a state of healthcare burnout and even collapse in some parts of the world with a shortage of primary healthcare facilities such as oxygen, medications, hospital beds, and ventilators. It is crucial to triage patients and admit only those who genuinely require expert supervision throughout their illness. Dendramis et al. gave a recommendation on hospital versus home care in patients of COVID-19 with BrS (Figure 3) [17,18]. 


\section{Cureus}

Hospital Admission Versus Home Care Management

in patients with

Brugada Syndrome and symptomatic COVID-19 Infection

Dendramis G. et al.

\begin{tabular}{|c|c|}
\hline $\begin{array}{l}\text { High risk } \\
\text { patients } \\
\text { Spontaneous abnormal Type } 1 \text { ECG pattern } \\
\text { Aborted sudden death } \\
\text { History of syncope due to sustained VT }\end{array}$ & $\begin{array}{l}\text { - Specialized hospital management with } \\
\text { additional precautions and monitoring for } \\
\text { arrhythmia. }\end{array}$ \\
\hline $\begin{array}{l}\text { Intermediate risk } \\
\text { patients } \\
\text { Spontaneous abnormal Type } 1 \text { EGG pattern } \\
\text { Asymptomatic patients }\end{array}$ & $\begin{array}{l}\text { Need to be observed with hospital } \\
\text { management until fever and major symptoms } \\
\text { resolves. }\end{array}$ \\
\hline $\begin{array}{l}\text { Low risk } \\
\text { patients } \\
\text { Not spontaneous abnormal Type } 1 \text { EEG pattern } \\
\text { Type } 2 \text { or } 3 \text { EEG pattern } \\
\text { Asymptomatic patients }\end{array}$ & $\begin{array}{l}\text { - May afford to self-isolate at home and } \\
\text { attendance at hospital should then be dictated } \\
\text { by syncope or other specific symptoms. }\end{array}$ \\
\hline \multicolumn{2}{|c|}{$\begin{array}{l}\text { FIGURE 3: Home care versus hospital admission in patients of COVID- } \\
19 \text { with BrS. }\end{array}$} \\
\hline \multicolumn{2}{|l|}{ Created with BioRender.com. } \\
\hline $\mathrm{BrS}=$ Brugada syndrome $; \mathrm{ECG}=$ elec & diogram; VT = ventricular tachycardia. \\
\hline
\end{tabular}

\section{Management}

With SCN5A gene mutations, sodium channels tend to malfunction at higher temperatures, unmasking BrS; therefore, pyrexia should be treated on a priority basis, and procedures causing pain that can increase parasympathetic tone should be done under proper analgesia. The management of $\mathrm{BrS}$ is focused on preventing its most feared outcome, i.e., SCD in a structurally normal heart. Electrolyte imbalances such as hypokalemia, hyperkalemia, and hypocalcemia should be managed promptly, and attention should be paid while prescribing drugs known to cause ventricular arrhythmias in patients with BrS. The first step of management entails educating the patient about triggers known to induce fatal arrhythmias. The first line of management remains an ICD for symptomatic patients who have a BrS pattern on ECG and mainly type 1 history of sudden cardiac arrest/SCD or unexplained syncope. ICD has shown to be superior to both pharmacotherapy and catheter ablation. Pharmacotherapy with antiarrhythmic drugs such as quinidine or amiodarone can be tried in patients with recurrent ventricular arrhythmia requiring multiple ICD shocks or in patients who have contraindications for ICD use (refusal by the patient for ICD implantation) and significant comorbidities with a reduced life expectancy [19].

For a patient with COVID-19 with BrS, the patient should be triaged for hospital admission or home care; before discharging, pyrexia should be controlled aggressively, and hospitalized patients should be monitored for arrhythmias and hemodynamic status. Patients of COVID-19 can be dehydrated and electrolyte imbalanced due to diarrhea, vomiting, and fever; fluid management should be done conservatively, and a passive leg raise test can be used to assess preload responsiveness. In patients admitted to the ICU, checking brain natriuretic peptide (BNP) and troponin levels and performing echocardiography are recommended to assess cardiac involvement.

\section{Conclusions}

Symptoms such as a syncopal episode with a recent history of fever could be a sign of a life-threatening underlying condition such as BrS. Pyrexia is a potential trigger for fatal cardiac arrhythmia such as VF in a previously asymptomatic patient. Taking a detailed history of previous episodes of syncope and family history of SCD plays an important role in diagnosing and risk stratifying these patients. Fever associated with the COVID-19 pandemic has unmasked various cases of BrS, and triaging patients based on their severity should be done to avoid the medical futility of critical healthcare facilities on a non-critical patient who can be managed at home.

\section{Additional Information}




\section{Disclosures}

Conflicts of interest: In compliance with the ICMJE uniform disclosure form, all authors declare the following: Payment/services info: All authors have declared that no financial support was received from any organization for the submitted work. Financial relationships: All authors have declared that they have no financial relationships at present or within the previous three years with any organizations that might have an interest in the submitted work. Other relationships: All authors have declared that there are no other relationships or activities that could appear to have influenced the submitted work.

\section{References}

1. Brugada J, Campuzano O, Arbelo E, Sarquella-Brugada G, Brugada R: Present status of Brugada syndrome: JACC state-of-the-art review. J Am Coll Cardiol. 2018, 72:1046-59. 10.1016/j.jacc.2018.06.037

2. Brugada P, Brugada J: Right bundle branch block, persistent ST segment elevation and sudden cardiac death: a distinct clinical and electrocardiographic syndrome: a multicenter report. J Am Coll Cardiol. 1992, 20:1391-6. 10.1016/0735-1097(92)90253-J

3. Probst V, Veltmann C, Eckardt L, et al.: Long-term prognosis of patients diagnosed with Brugada syndrome: results from the FINGER Brugada Syndrome Registry. Circulation. 2010, 121:635-43. 10.1161/CIRCULATIONAHA.109.887026

4. Kusano KF, Taniyama M, Nakamura K, et al.: Atrial fibrillation in patients with Brugada syndrome: relationships of gene mutation, electrophysiology, and clinical backgrounds. J Am Coll Cardiol. 2008, 51:1169-75. 10.1016/i.jacc.2007.10.060

5. BrugadaDrugs.org. Safe drug use and the Brugada syndrome. (2020). https://www.brugadadrugs.org.

6. Chang D, Saleh M, Garcia-Bengo Y, Choi E, Epstein L, Willner J: COVID-19 infection unmasking Brugada syndrome. HeartRhythm Case Rep. 2020, 6:237-40. 10.1016/j.hrcr.2020.03.012

7. Choi NH, Silver ES, Fremed M, Liberman L: COVID-19 reveals Brugada pattern in an adolescent patient Cardiol Young. 2020, 30:1735-7. 10.1017/S1047951120002619

8. Kim MS, Frenkel SL, Hayden SR: Fever unmasking Brugada-pattern electrocardiogram in patient with coronavirus disease 2019 (COVID-19). J Emerg Med. 2020, 59:439-41. 10.1016/j.jemermed.2020.06.053

9. Maglione TJ, Aboyme A, Ghosh BD, Bhatti S, Kostis WJ: Electrical storm in a febrile patient with Brugada syndrome and COVID-19 infection. HeartRhythm Case Rep. 2020, 6:676-9. 10.1016/j.hrcr.2020.07.008

10. Pasquetto G, Conti GB, Susana A, Leone LA, Bertaglia E: Syncope, Brugada syndrome, and COVID-19 lung disease. J Arrhythm. 2020, 36:768-70. 10.1002/joa3.12375

11. Tsimploulis A, Rashba EJ, Rahman T, Almasry IO, Singh A, Fan R: Medication unmasked Brugada syndrome and cardiac arrest in a COVID-19 patient. HeartRhythm Case Rep. 2020, 6:554-7. 10.1016/j.hrcr.2020.05.012

12. Behr ER, Savio-Galimberti E, Barc J, et al.: Role of common and rare variants in SCN10A: results from the Brugada syndrome QRS locus gene discovery collaborative study. Cardiovasc Res. 2015, 106:520-9. 10.1093/cvr/cvv042

13. Wilde AA, Antzelevitch C, Borggrefe M, et al.: Proposed diagnostic criteria for the Brugada syndrome: consensus report. Circulation. 2002, 106:2514-9. 10.1161/01.cir.0000034169.45752.4a

14. Veltmann C, Wolpert C, Sacher F, et al.: Response to intravenous ajmaline: a retrospective analysis of 677 ajmaline challenges. Europace. 2009, 11:1345-52. 10.1093/europace/eup189

15. Priori SG, Wilde AA, Horie M, et al.: HRS/EHRA/APHRS expert consensus statement on the diagnosis and management of patients with inherited primary arrhythmia syndromes: document endorsed by HRS, EHRA, and APHRS in May 2013 and by ACCF, AHA, PACES, and AEPC in June 2013. Heart Rhythm. 2013, 10:193263. 10.1016/i.hrthm.2013.05.014

16. Gourraud JB, Barc J, Thollet A, Le Marec H, Probst V: Brugada syndrome: diagnosis, risk stratification and management. Arch Cardiovasc Dis. 2017, 110:188-95. 10.1016/j.acvd.2016.09.009

17. Dendramis G, Brugada P: Intensive care and anesthetic management of patients with Brugada syndrome and COVID-19 infection. Pacing Clin Electrophysiol. 2020, 43:1184-9. 10.1111/pace.14044

18. Pappone C, Santinelli V: Brugada syndrome: progress in diagnosis and management . Arrhythm Electrophysiol Rev. 2019, 8:13-8. 10.15420/aer.2018.73.2

19. Al-Khatib SM, Stevenson WG, Ackerman MJ, et al.: 2017 AHA/ACC/HRS guideline for management of patients with ventricular arrhythmias and the prevention of sudden cardiac death: a report of the American College of Cardiology/American Heart Association Task Force on Clinical Practice Guidelines and the Heart Rhythm Society. J Am Coll Cardiol. 2018, 72:e91-e220. 10.1016/j.jacc.2017.10.054 